Effect of coi I conf i gur at i on par anet ers on the mechani cal behavi or of the superconduct ing magnet system i $n$ the hel i cal fusi on react or FFHR

\begin{tabular}{|l|l|}
\hline $\begin{array}{l}\text { j our nal or } \\
\text { publ i cat i on } \mathrm{titl} \text { e }\end{array}$ & Fusi on Engi neer i ng and Desi gn \\
\hline vol une & 146 \\
\hline number & Part A \\
\hline page r ange & $586-589$ \\
\hline year & $2019-01$ \\
\hline NAl S & 11899 \\
\hline URL & ht t p: //hdl . handl e. net /10655/00012720 \\
\hline
\end{tabular}




\title{
Effect of coil configuration parameters on the mechanical behavior of the superconducting magnet system in the helical fusion reactor FFHR
}

\author{
Hitoshi Tamura ${ }^{a}$, Takuya Goto ${ }^{a}$, Nagato Yanagi ${ }^{a}$, Junichi Miyazawa ${ }^{a}$, Teruya Tanaka ${ }^{a}$, \\ Akio Sagara $^{a}$, Satoshi Ito $^{b}$, and Hidetoshi Hashizume ${ }^{b}$ \\ ${ }^{a}$ National Institute for Fusion Science, Toki, Japan \\ ${ }^{b}$ Tohoku University, Sendai, Japan
}

\begin{abstract}
FFHR-d1A and $\mathrm{c} 1$ are the conceptual design of a helical fusion reactor. The positional relationship among superconducting coils, a pair of helical coils with two sets of vertical-field coils, are observed to be similar in both type of FFHR. Such a relation of coil configuration is based on the coil configuration of the Large Helical Device (LHD), which has been designed and constructed at the National Institute for Fusion Science. There is increasing demand to achieve an optimized coil configuration to anticipate improvements in plasma-confinement conditions. In this study, the structural design of FFHR based on the fundamental set of parameters of coil configuration is depicted, which satisfies the soundness of the structure. Further, the effects of the coil configuration parameters on the stress distributions are investigated. An effect of radius of curvature on a winding scheme of the helical coil is also discussed.
\end{abstract}

Keywords: helical fusion reactor, large helical device, superconducting magnet, multiscale analysis, structural design

\section{Introduction}

FFHR is a conceptual design for a helical fusion reactor being developed by the National Institute for Fusion Science (NIFS) in collaboration with several universities [1-3]. Several FFHR designs have been investigated; for instance, FFHR-d1A is a self-ignition demonstration reactor that operates at a magnetic-field intensity of $4.7 \mathrm{~T}$ and has a major radius of $15.6 \mathrm{~m}$; FFHRc1 is a compact-type sub-ignition reactor intended to achieve steady electrical self-sufficiency and with a higher magnetic-field intensity of $7.3 \mathrm{~T}$ and a smaller major radius of $10.92 \mathrm{~m}$.

The positional relationship among superconducting coils, a pair of helical coils (HCs) with two sets of vertical field coils (VFCs), are observed to be similar in both type of FFHR. This relation is based on the Large Helical Device (LHD) coil configuration [4]. This configuration is defined using an aspect ratio, the pitch and modulation parameter of the $\mathrm{HC}$, the number and geometric position of the VFCs, and so on.

There is increasing demand for achieving an optimized coil configuration to anticipate improvements in plasma-confinement conditions. However, few investigations have studied the effect of coil configuration (including the coil-support structure) upon the mechanical behaviors of coil systems. Furthermore, since the electromagnetic (EM) force induced by a magnet system is proportional to the square of the magnetic-fieldintensity ratio, the stress on the magnet system can be extremely severe under increases of the magnetic-field intensity. According to the latest structural analysis of the FFHR-d1A magnet system, the maximum von Mises stress in the coil-support structure is $764 \mathrm{MPa}$ at a typical thickness of $250 \mathrm{~mm}$ [5]. The coil-support structure of the reduced-size FFHR-c1 needs to be modified, including in terms of its thickness; otherwise, the stress level will exceed the allowable limit of a structural material.

In this study, the structural design of FFHR-c1 is presented based on the fundamental set of coilconfiguration parameters, satisfying the soundness of the structure. Furthermore, the effects of the coil-pitchmodulation parameter upon the stress/strain distributions are investigated. The effect of the HC-winding scheme is also discussed.

\section{Fundamental design of FFHR-c1}

\subsection{Coil configuration}

The trajectory of the current center of the $\mathrm{HC}$ is defined by the pseudo toroidal coordinate with major / minor radii of $R_{c} / a_{c}$, shown in figure 1 as

$$
\theta=-\left\{\frac{m}{l} \varphi+\alpha \sin \left(\frac{m}{l} \varphi\right)\right\}
$$

Here, $\theta, \varphi, l, m$, and $\alpha$ are the poloidal angle, toroidal angle, pole number, pitch number, and pitch-modulation parameter, respectively. Figure 2 shows the trajectory of the $\mathrm{HC}$ current center with respect to various coil-pitchmodulation parameters $\alpha$. When $\alpha$ is positive, the $\mathrm{HC}$ has a shape that is inclined with respect to the vertical side on the outboard of the torus region, and to the horizontal side on the inboard. In the negative- $\alpha$-value case, the $\mathrm{HC}$ tends to incline to the opposite side. For $\alpha=0$, the coil-winding center is located at poloidal angles of $90^{\circ}(\mathrm{HC} 2)$ and $270^{\circ}$ (HC1) where the toroidal angle is $18^{\circ}$. It appears inside the torus when $\alpha$ is positive but comes to a position close to the outside of the torus when $\alpha$ is negative, as shown in figure 2. LHD and the fundamental design of FFHR-c1 adopted $\alpha=0.1$ [4]. 


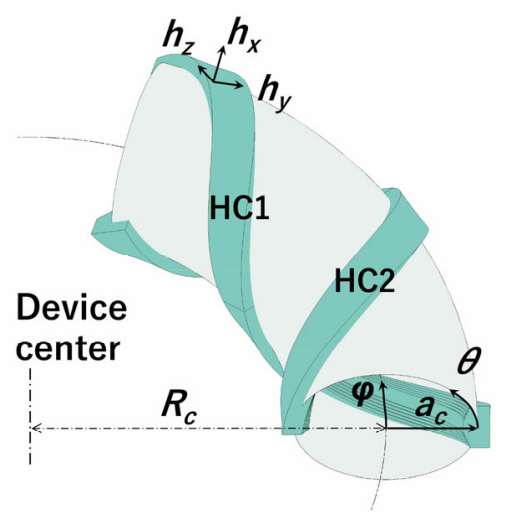

Fig. 1. Definition of the pseudo toroidal coordinate indicated in eq. (1) and the HC parameter.
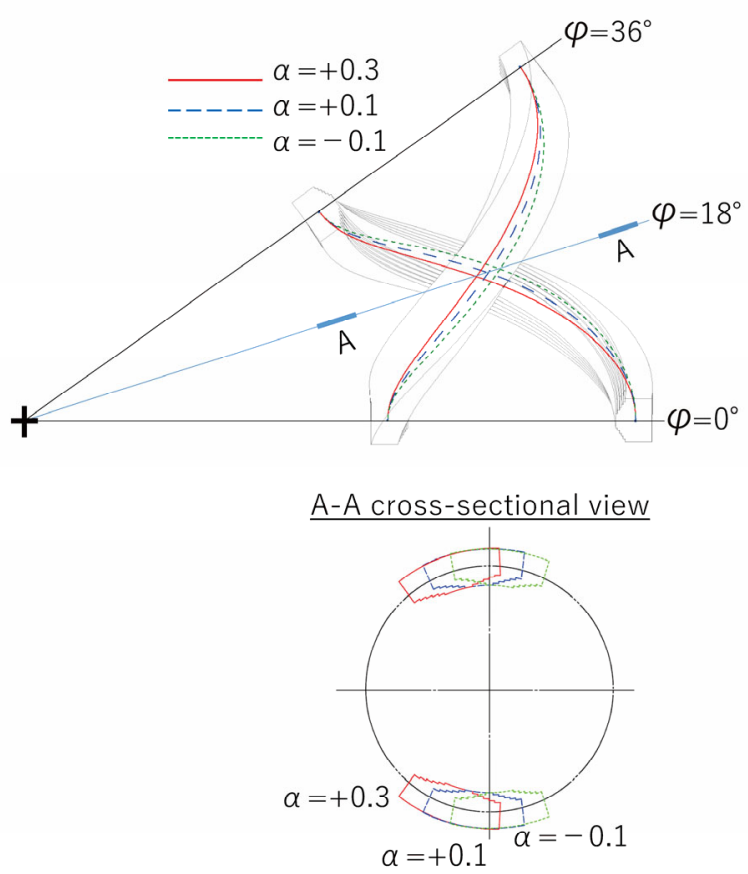

Fig. 2. Trajectories of the current center of the HCs in accordance with the pitch-modulation parameter $\alpha$.

\subsection{Coil layout and support structure}

The coil locations of FFHR-d1A and $\mathrm{c} 1$ are similar. Based on the coil geometry of FFHR-d1A, the fundamental layout of the coils and the support structure for FFHR-c1 was modeled as shown in figure 3. In this model, a supplemental helical coil, named the NITA coil, was added outside of the HC. The distance between the plasma surface and the bottom of the $\mathrm{HC}$ can be enlarged by adding the NITA coil [6]. This space is used for the invessel components, coil case, and gap for the adiabatic vacuum. The cross-sectional shape of the HC was carefully chosen to have appropriate clearance between the $\mathrm{HC}$ and in-vessel components like a shielding / breeding blanket. The basic thickness of the coil-support structure was assumed to be $200 \mathrm{~mm}$, which is $250 \mathrm{~mm}$ in the $\mathrm{d} 1 \mathrm{~A}$. The superconductor used in the $\mathrm{c} 1 \mathrm{was}$ assumed to have a similar structure to that of the $\mathrm{d} 1 \mathrm{~A}[7,8]$ and the size was reduced to $43.32 \mathrm{~mm} \times 43.32 \mathrm{~mm}$. The magnetomotive force (coil current) in each coil and the current density are also shown in table 1 .

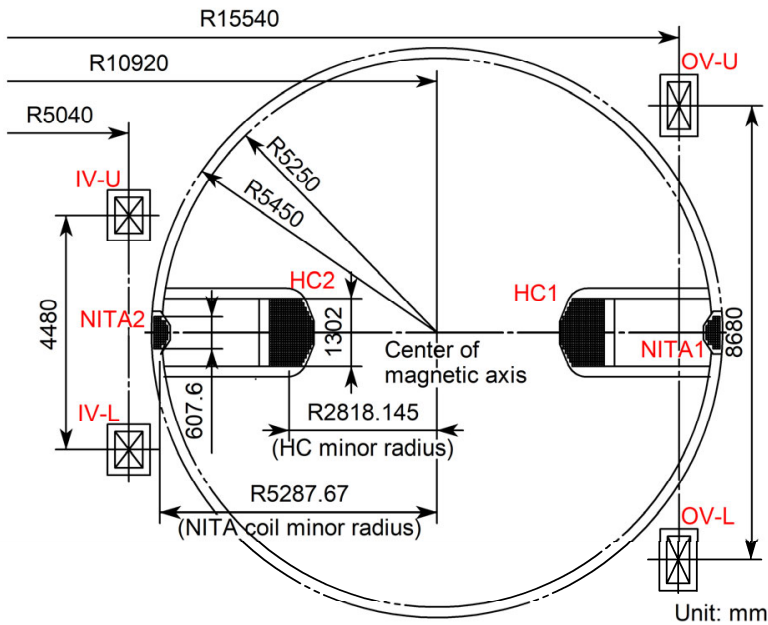

Fig. 3. Drafting sketch of the coil and support structure. IV$\mathrm{U} / \mathrm{L}$ and $\mathrm{OV}-\mathrm{U} / \mathrm{L}$ represent the inner (upper/lower part) and outer (upper/lower part) VFC, respectively.

Table 1. Specification of each coil in FFHR-c1 assumed in the analyses for all case of $\alpha$.

\begin{tabular}{lcccc}
\hline & HC & NITA & OVF & IVF \\
\hline $\begin{array}{l}\text { Magnetomotive } \\
\text { force (MA) }\end{array}$ & 45.99 & -6.132 & -21.51 & 24.52 \\
$\begin{array}{l}\text { Number of turns } \\
\begin{array}{l}\text { Current density } \\
\left(\mathrm{A} / \mathrm{mm}^{2}\right)\end{array}\end{array}$ & 510 & 68 & 208 & 210 \\
\hline
\end{tabular}

\section{Analyses}

\subsection{Electromagnetic (EM) force}

The EM force of the $\mathrm{HC}$ can be divided into two components in the hoop and overturning directions with respect to the coil-winding direction. Let the coordinate axes, $h_{x}$ and $h_{y}$, be chosen such that they are in the crosssectional plane of the $\mathrm{HC}$ and their directions coincide with the coil height and width, as shown in figure 1. The $h_{z}$ axis is perpendicular to the coil cross section and is consistent with the winding direction of the coil, i.e., the current-flow direction. The hoop force, $F_{a}$, and the overturning force, $F_{b}$, can be defined that the force components of $h_{x}$ and $h_{y}$ directions, respectively. For the VFCs, a force in the radial direction indicates the hoop force, $F_{r}$, and in the vertical direction indicates an attraction force, $F_{z}$, or a repulsion force, $-F_{z}$. Since the $\mathrm{HC}$ has a cyclic symmetry of $m$, the first $2 \pi /(\mathrm{m} / \mathrm{l})$ should be considered. Here, we set the parameter $l=2, m=10$, according to the design of FFHR-c1 [3]. In this case, it has a cyclic symmetry of 10 and the section of $2 \pi / 5=72^{\circ}$ should be taken into account.

The three-dimensional magnetic-field distribution induced by the coils was calculated using the finiteelement method. The coils and surrounding space were modeled as a vector-potential element in ANSYS ${ }^{\circledR}$. All 
material was assumed to be non-magnetic. Consequently, the magnetic-field distribution was obtained and an overall EM force was calculated by accumulating a magnetic field at every single superconductor position and multiplying them by the current of the superconductor. The overall EM forces on the HC1 in the cases of $\alpha=-0.1$, 0.1 , and 0.3 are shown in figure 4. The EM force for $\mathrm{HC} 2$ can be obtained by shifting the vertical scale $36^{\circ}$ forward in the result graph for $\mathrm{HC} 1$. The maximum $\mathrm{EM}$ hoop force, $\mathrm{F}_{\mathrm{a}}$, is $140 \mathrm{MN} / \mathrm{m}$ in the case of $\alpha=-0.1$. The maximum EM overturning force $F_{b}$ is approximately $\pm 30 \mathrm{MN} / \mathrm{m}$, and the average is generally zero for any $\alpha$ case. Even with slight differences in $\alpha$, there is a big difference in maximum value and amplitude. When compared with $\alpha=$ $-0.1,0.1,0.3$, the maximum value of $\mathrm{F}_{\mathrm{a}}$ decreases and the minimum value increases as $\alpha$ increases. Furthermore, the change of $F_{a}$ with respect to the toroidal angle decreases as $\alpha$ increases. In the case of 0.1 , the absolute value of the overturning force, $\mathrm{Fb}$, is small. Compared with the cases of $\alpha=-0.1$ and 0.3 , the overturning direction is reversed.

Figure 5 shows the EM force on the NITA1, IV-U, and OV-U coils. The EM force for NITA2 is obtained from NITA1 as well as the same manner with that for HC2 from $\mathrm{HC} 1$. Although there is some peak shift and change in the magnitudes of EM forces, the effect of $\alpha$ is small compared with the $\mathrm{HC}$ case. The maximum magnetic fields on the coils for $\alpha=0.1$ are HC: 18.9 T, NITA: 10.9 $\mathrm{T}$, inner-VFC: $18.5 \mathrm{~T}$, outer-VFC: $12.5 \mathrm{~T}$. The maximum magnetic field decreases as $\alpha$ increases in case of $-0.1 \leq$ $\alpha \leq 0.2$. The maximum magnetic field in case of $\alpha=0.3$ is slightly greater than that in case of $\alpha=0.2$. There is little difference in stored energy, however, the tendency of change is similar to that of the maximum magnetic field

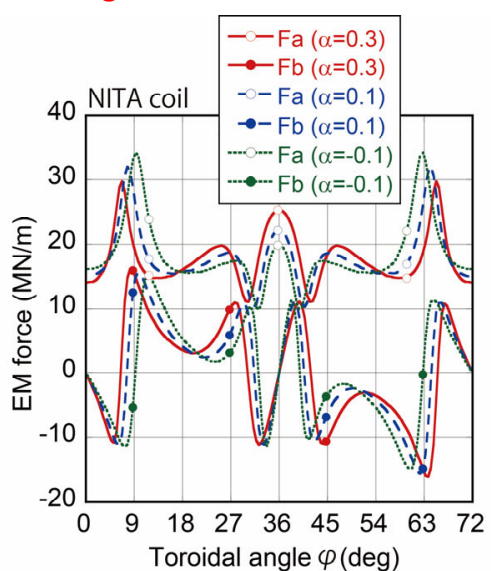

as shown in table 2. The maximum magnetic field appears at the inboard side of the torus when $\alpha=-0.1$, and gradually shift to the top / bottom region of the torus. A vertical component of the outer magnetic field at the top / bottom region is higher than that at the inboard region. An effect of vertical magnetic field becomes larger when $\alpha \geq 0.3$, and it leads such the tendency of the maximum magnetic field.

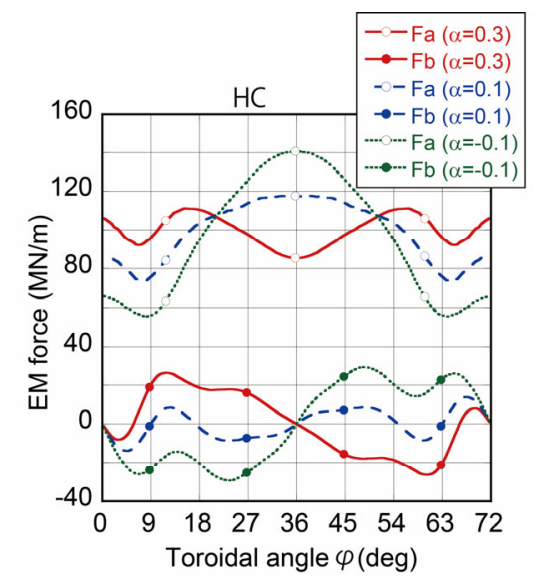

Fig. 4. Overall EM forces on the HC1.

Table 2. Effect of the coil-pitch-modulation parameter on the magnetic condition.

\begin{tabular}{lccccc}
\hline \multicolumn{1}{c}{$\alpha$} & -0.1 & 0 & 0.1 & 0.2 & 0.3 \\
\hline $\begin{array}{l}\text { Maximum } \\
\text { magnetic field (T) }\end{array}$ & 19.4 & 19.2 & 18.9 & 18.7 & 18.8 \\
$\begin{array}{l}\text { Stored energy } \\
\text { (GJ) }\end{array}$ & 157.5 & 157.0 & 156.8 & 156.7 & 156.9 \\
\hline
\end{tabular}

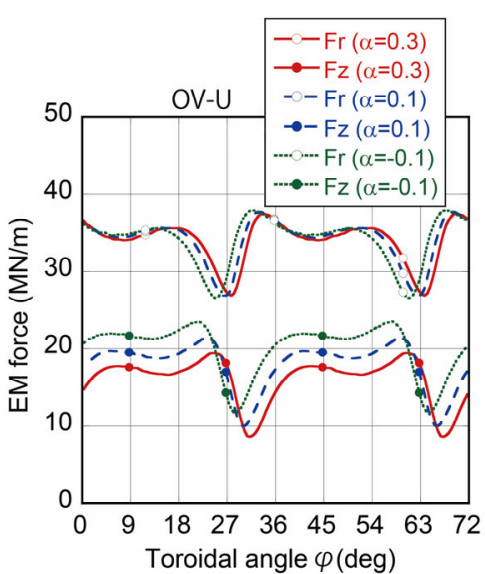

Fig. 5. Overall EM forces on HC1, NITA1, IV-U, and OV-U.

\subsection{Stress and deformation}

Based on the fundamental design of the support structure for FFHR-d1A [5], the shape of that of FFHRc1 was made by reducing its size and adopting a largecurrent high-temperature superconductor (HTS) [9]. The support structure was assumed to be made of SS316LN with a basic thickness of $200 \mathrm{~mm}$. The design was rearranged until the maximum von Mises stress in the structure fell within an acceptable level. The physical properties of the HTS were estimated using multiscalehomogenization analysis [7]. The force obtained in the EM analysis was assigned to the appropriate nodes in the model.

Figure 6 shows resultant of the structural analysis for $\alpha=-0.1,0.1$, and 0.3 . Focusing on several high-stress areas, the effect of $\alpha$ is investigated. As $\alpha$ increases, the maximum von Mises stress at the $\mathrm{HC}$ case and the support arm increase, and stress at the inner-VFC coil slightly 
decreases. Since the EM force is a body force, and the volume of $\mathrm{HC}$ at the outboard side is larger than that of the inboard side, the amount of EM force on the $\mathrm{HC}$ at the outboard side region considered to be dominant. The EM force at the outboard side increases as $\alpha$ increases as shown in figure 4. Furthermore, high stress is likely to appear at the inboard of torus when the torus is subjected to an uniform pressure. The analytic results reflected these effects.

The soundness of the support structure will be guaranteed if a high-strength material like SS316LNH used in ITER [10] is chosen. The effect of $\alpha$ upon the stress in the coil-support structure is summarized in table 2. The maximum deformation was approximately $16 \mathrm{~mm}$ and it appeared at the outer-VFC region in all $\alpha$ cases.

\section{Discussion}

One critical issue related to the helical reactor is a winding procedure for the coils. The curvature $\kappa$ of the

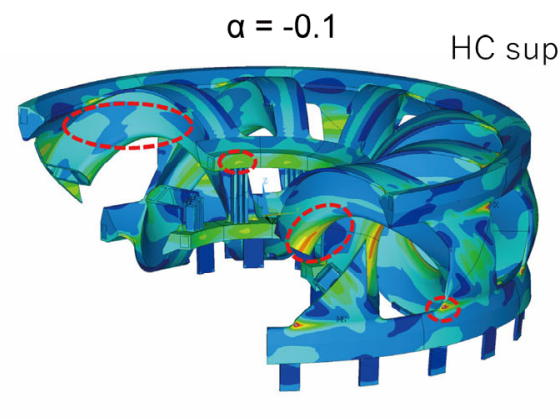

$\alpha=+0.1$

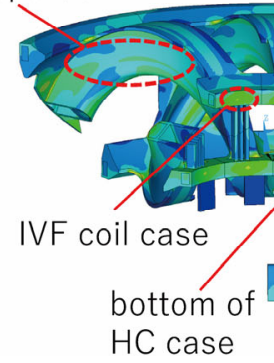

trajectory curve $r(s)$ is calculated from following equation:

$$
\kappa=\left\|\frac{d^{2} \boldsymbol{r}(s)}{d s^{2}}\right\|
$$

where $s$ is arc length. The radius of curvature, $1 / \kappa$, changes through the toroidal angle, as shown in figure 7 , and changes according to the pitch-modulation parameter $\alpha$. For $\alpha=-0.1$, the radius of curvature varies form 3.92 $\mathrm{m}$ to $4.94 \mathrm{~m}$, and the radius at the inboard of the torus range is smaller than that at the outboard. On the other hand, it varies from $3.95 \mathrm{~m}$ to $6.17 \mathrm{~m}$, and the radius at the inboard is larger than that of the outboard for $\alpha=0.3$. The change and tendency are moderate for $\alpha=0.1$. In a point of $\mathrm{HC}$ winding with thick rigid conductor, it is considered that the moderate change of the curvature through the entire winding path is preferable.

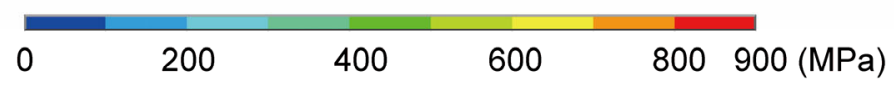

Fig. 6. Von Mises-stress distribution of the coil-support structure based on FFHR-c1.

Table 2. Effect of the coil-pitch-modulation parameter upon the von Mises stress level at a specific region of the coil-support structure. (unit: $\mathrm{MPa}$ )

\begin{tabular}{lccccc}
\hline \multicolumn{1}{c}{$\alpha$} & -0.1 & 0 & 0.1 & 0.2 & 0.3 \\
\hline HC case & 761 & 773 & 787 & 804 & 822 \\
HC support arm & $\sim 300$ & $\sim 310$ & $\sim 340$ & $\sim 390$ & $\sim 450$ \\
Inner-VFC case & $\sim 510$ & $\sim 510$ & $\sim 500$ & $\sim 500$ & $\sim 490$ \\
Outer port (peak) & 1060 & 1010 & 995 & 979 & 1070 \\
\hline
\end{tabular}

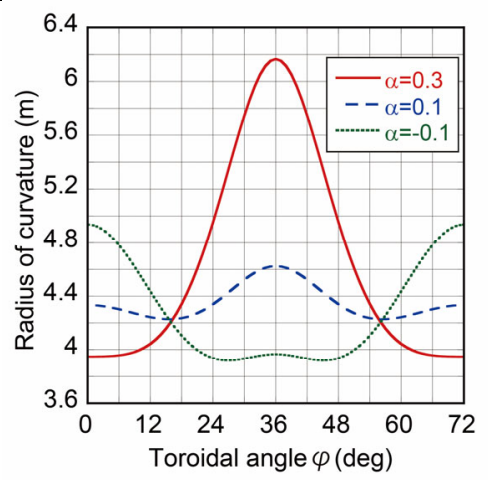

Fig. 7. Radius of curvature of the HC current center.
The effects of the coil-pitch-modulation parameter, $\alpha$, of a helical fusion reactor were investigated and robust designs of the helical coil and its support structure were demonstrated. Consequently, the following results were obtained. 1) Maximum magnetic field decreases as $\alpha$ increases in case of $-0.1 \leq \alpha \leq 0.2$. The maximum magnetic field in case of $\alpha=0.3$ is slightly greater than that in case of $\alpha=0.2$. 2) Maximum stress on the HC increases as $\alpha$ increases. 3) Radius of curvature changes greatly depending on $\alpha$. 4) $\alpha \approx 0.1$ is reasonable for the specific-coil layout of FFHR-c1 from the standpoint of support structure and coil winding.

\section{Acknowledgments}

The present study has been conducted under the grant from the NIFS (No. UFFF031). This work was supported by JSPS KAKENHI Grant Numbers 26220913, 16K06943, and 17H03507.

\section{Conclusions}




\section{References}

[1] A. Sagara, et al., Review of stellarator/heliotron design issues towards MFE DEMO, Fusion Eng. Des. 85 (2010) 1336-1341.

[2] A. Sagara, et al., Design activities on helical DEMO reactor FFHR-d1, Fusion Eng. Des. 87 (2012) 594-602.

[3] A. Sagara, et al., Helical reactor design FFHR-d1 and c1 for steady state DEMO, Fusion Eng. Des. 89 (2014) 2114-2120.

[4] O. Motojima, et al., Physics and engineering design studies on the Large Helical Device, Fusion Eng. Des. 20 (1993) 314.

[5] H. Tamura et al., Design modification of structural components for the helical fusion reactor FFHR-d1 with challenging options, Fusion Eng. Des. 124 (2017) 605609.

[6] N. Yanagi, et al., NITA coil - innovation for enlarging the blanket space in the helical fusion reactor, Plasma Fusion Res. 11 (2016) 2405034.

[7] H. Tamura, et al., Multiscale stress analysis and 3D fitting structure of superconducting coils for the helical fusion reactor, IEEE Trans. Appl. Supercond. 26 (2016) 4202405.

[8] H. Tamura et al., Design Status of the Structural Components of the Helical Fusion Reactor FFHR-d1, Plasma Fusion Res 11 (2016) 2405061.

[9] N. Yanagi, et al., Design and development of hightemperature superconducting magnet system with jointwinding for the helical fusion reactor, Nucl. Fusion 55 (2015) 053021.

[10] ITER structural design criteria for magnet components (SDC-MC), Inst. Tech. Educ. Res. (ITER), Naka, Japan, N11 FDR 5001-07-05 R 0.1 (2001). 